\title{
Desenvolvimento e Validação do Sistema de Observação do Clima de Aula em Aulas de Grupo de Fitness - Aplicação Piloto em Idosos
}

\section{Development and Validation of the Class Climate Observation System in Group Fitness Classes - Pilot Application with the Elderly}

\section{Desarrollo y Validación del Sistema de Observación del Clima en Clases de Grupo de Fitness - Aplicación Piloto en Ancianos}

\author{
Dias, I1, Franco, S1,2, Ramos, L1,2, Simões, V1,2 \\ ${ }_{1}$ Escola Superior de Desporto de Rio Maior - Instituto Politécnico de Santarém; \\ 2Centro de Investigação em Qualidade de Vida (CIEQV)
}

\section{RESUMO}

O presente estudo teve como principal objetivo desenvolver e validar um Sistema de Observação do Clima de Aula em Aulas de Grupo de Fitness (SOCA-AGF) bem como realizar uma aplicação piloto do mesmo. Participaram no estudo 6 instrutoras de 2 atividades (hidroginástica e localizada), em população idosa, para testar a funcionalidade do instrumento. Verificou-se que o SOCA-AGF, constituído por 2 dimensões, 36 categorias e 8 subcategorias, apresentou fiabilidade e validade. Os resultados obtidos indicam que o comportamento observável dos instrutores de fitness, em aulas de grupo, poderá ser codificado recorrendo a este instrumento. Concluiu-se que o SOCA-AGF regista aspetos fundamentais do comportamento dos instrutores de fitness que influencia o clima em aulas de grupo e, como tal, poderá ser utilizado para estudar aspetos relevantes no âmbito do comportamento que influencia o clima de aula em diferentes atividades de grupo, no contexto do fitness, e em diferentes tipos de população.

Palavras chave: observação, comportamento do instrutor, clima de aula, fitness.

\begin{abstract}
The main target of this study was to develop and validate an observation system of the climate in group fitness classes (SOCA-AGF), as well to carry out a pilot implementation. A sample of 6 instructors from 2 activities (aquafitness and resistance training) with elderly people tested the functionality of the instrument. The observation system SOCAAGF, which has 2 dimensions, 36 categories and 8 subcategories, showed reliability and validity. The results indicate that the observable behaviour of fitness instructors in group classes can be codified by using this instrument. We concluded that the SOCA-AGF shows fundamental fitness instructor behaviour aspects that influence the climate in group fitness classes, therefore it can be used to study relevant aspects of the behavior that influences the class climate in different group activities, in the context of fitness and with different types of population.
\end{abstract}

Keywords: observation, instructor's behaviour, class climate, fitness. 


\section{Dias, I., Franco, S., Ramos, L., Simões, V.}

\section{RESUMEN}

El objetivo del estudio fue desarrollar y validar un Sistema de Observación del Clima de Clase en Clases de Grupo de Fitness (SOCA-AGF), así como realizar una aplicación piloto del mismo. Para esta aplicación piloto se utilizó una muestra de 6 instructores de 2 actividades (aquafitness a y localizada), en ancianos, para probar la funcionalidad del instrumento. Se verificó que el SOCA-AGF, constituido por 2 dimensiones, 36 categorías y 8 subcategorías, presentó fiabilidad y validez. Los resultados obtenidos indican que el comportamiento observable de los instructores, en clases de grupo, puede ser codificado recurriendo a este instrumento. Se concluyó que el SOCA-AGF registra aspectos fundamentales del comportamiento de los instructores de fitness que influye en el clima de clases de grupo y, como tal, podrá ser utilizado para estudiar aspectos relevantes en el ámbito del comportamiento que influye en el clima de clase en diferentes actividades de grupo en el contexto del fitness y en diferentes tipos de población.

Palabras clave: observación, comportamiento del instructor, clima de clase, fitness

\section{INTRODUÇÃO}

De acordo com a European Health \& Fitness Association (2012), atualmente designada por EuropeActive, para além da elevada procura pelas atividades de fitness, o número de instalações deste género, assim como o número de indivíduos praticantes deste tipo de atividades, cresceu de forma acentuada. O grande desafio colocado a organizações que prestam serviços na área do fitness prende-se essencialmente, segundo Gomes, Chagas e Mascaranhas (2010), com a atração de novos praticantes, bem como com a sua permanência. Loughead, Colman e Carron (2001) afirmam que alguns dos comportamentos dos instrutores de fitness, designadamente os comportamentos associados à criação de um clima positivo, proporcionam um ambiente que promove a adesão e consequente permanência dos praticantes. De acordo com Franco e Simões (2017), considerar-se-á particularmente importante que os instrutores tenham um comportamento que vá ao encontro do que os praticantes preferem, para que seja mantida a adesão à prática de exercício, evitando o abandono. É importante que a satisfação dos praticantes seja uma preocupação constante dos instrutores, pois, como constataram Collishaw, Dyer e Boies (2008), existe uma relação positiva entre a satisfação dos praticantes e a consequente fidelização para com o instrutor de fitness, quando este comunica com entusiasmo.

Vários são os estudos onde é referida a importância que o instrutor pode ter na apresentação de um serviço de qualidade dos ginásios, na satisfação, motivação, prazer, adesão e fidelização dos praticantes relativamente à prática de exercício (Bray, Gyurcsik, Culos-Reed, Dawson \& Martin, 2001; Carron,
Hausenblas \& Estabrooks, 1999; Carron, Hausenblas \& Mack, 1996; Lippke, Knäuper \& Fuchs, 2003; Loughead, Colman \& Carron, 2001; Loughead \& Carron, 2004; Murray \& Howat, 2002; Papadimitriou \& Karteroliotis, 2000; Rodrigues et al., 2018; Rodrigues et al., 2019; Theodorakis, Alexandris, Rodriguez \& Sarmento, 2004; Wininger, 2002).

A observação sistemática é reconhecida por vários autores como sendo um efetivo instrumento de pesquisa na descrição quantitativa dos comportamentos do treinador de desporto e/ou do professor de Educação Física (Anguera, Blanco, Losada \& Hernández-Mendo, 2000; Mars, 1989; Sarmento, Veiga, Rosado, Rodrigues \& Ferreira, 1998). O comportamento pedagógico dos instrutores de fitness, nas aulas de grupo, tem vindo a ser uma preocupação por parte de vários investigadores, tal como mencionado numa revisão sistemática desenvolvida por Rodrigues et al. (2018). Neste sentido, alguns instrumentos têm vindo a ser desenvolvidos no contexto do fitness nomeadamente: o Sistema de Observação do Comportamento dos Instrutores de fitness - SOCIF (Franco, Rodrigues \& Balcells, 2008) que permite analisar de forma geral o comportamento pedagógico dos instrutores de fitness em aulas de grupo; o Sistema de Observação de Feedback de Instrutores de fitness em Aulas de Grupo - SOFIF-AG (Franco \& Simões, 2017), que permite analisar o comportamento de feedback realizado pelos instrutores de fitness em aulas de grupo; o Sistema de Observação da Comunicação Proxémica do Instrutor de fitness, em Aulas de Grupo - SOPROX-fitness (Alves et al., 2013), que permite analisar a comunicação proxémica dos instrutores de aulas de grupo e o Sistema de Observação da Comunicação 


\section{Desenvolvimento e Validação do SOCA-AGF - Aplicação Piloto em Idosos}

Cinésia - SOCIN- fitness (Alves et al., 2014) que permite analisar a comunicação cinésia dos instrutores de fitness em aulas de grupo. Relativamente ao comportamento do instrutor de fitness foi recentemente validado para português o questionário relativo à satisfação e frustração das necessidades dos instrutores de fitness (Rodrigues et al., 2019). No que diz respeito ao comportamento acerca do clima de aula foi encontrado um instrumento de observação denominado Sistema de Observação do Clima de Aula de Sarmento, Veiga, Rosado, Rodrigues e Ferreira (1998), cujo objetivo é avaliar variáveis/comportamentos do professor associados à criação de um clima de aula positivo, e que já foram utilizados em alguns estudos no contexto do fitness, embora com adaptações (Franco, Simões, Alves, Vidal \& Silva, 2010). O referido instrumento é constituído por 17 categorias de análise representativas de alguns dos comportamentos mais provavelmente responsáveis pela criação de um clima de aula positivo.

Para analisar o comportamento de determinado indivíduo, utilizando a observação como método, torna-se necessário utilizar instrumentos adaptados e adequados às necessidades do contexto e da pesquisa. Ao nível do comportamento, existe uma diversidade de situações que são suscetíveis de serem sistematicamente observadas, como tal torna-se importante prescindir de instrumentos estandardizados, desenvolvendo desta forma instrumentos de acordo com os contextos específicos que se pretendem estudar (Prudente, Garganta \& Anguera 2004; Sarmento, 2004).

Assim sendo o objetivo deste estudo passa por desenvolver e validar um Sistema de Observação do Clima de Aula, em Aulas de Grupo de Fitness (SOCAAGF), que permita codificar os comportamentos dos instrutores, ao nível do clima de aula, e realizar uma aplicação piloto do mesmo, nas atividades de localizada e hidroginástica, em população idosa.

\section{MATERIAL E MÉTODOS}

\section{Desenvolvimento e Validação do SOCA-AGF}

Para desenvolver e validar o SOCA-AGF foram seguidos pressupostos recomendados por diversos autores e utilizados para a validação de instrumentos similares (Anguera, Blanco, Losada \& HernándezMendo, 2000; Brewer \& Jones, 2002; Anguera, 2003; Prudente, Garganta \& Anguera, 2004, Bakeman,
Quera \& Gnisci, 2009; Hernández-Mendo, Martínez \& Morales-Sánchez, 2010; Gilbert, Trudel, Gaumond \& Larocque, 1999; Costa, Garganta, Greco, Mesquita \& Maia, 2011; Franco, 2009; Alves et al., 2013; Alves et al., 2014; Alves et al., 2015; Losada \& Manolov, 2015; Anguera et al., 2017; Franco \& Simões, 2017; Chacón-Moscoso et al., 2018). Optou-se pelo desenvolvimento de um sistema de observação misto de formatos de campo e categorias, tal como na maioria dos estudos de observação relacionados com a área do desporto (Anguera, 2003; Anguera \& Blanco, 2003, 2005; Anguera, Magnusson \& Jonsson, 2007; Anguera \& Hernández-Mendo, 2013). Desta forma, para o desenvolvimento e validação do SOCAAGF teve-se como base o Sistema de Observação de Clima de Aula desenvolvido e validado por Sarmento et al. (1998), para as aulas de Educação Física. Em seguida os procedimentos foram divididos em 5 fases, nomeadamente:

Fase 1: Treino dos observadores relativamente ao sistema de observação original

$\mathrm{O}$ treino dos observadores tem como objetivo assegurar que estes conhecem o instrumento existente na totalidade, assim como compreendem todas as suas terminologias e conceitos. Indo ao encontro das sugestões apresentadas por Carreiro da Costa (1988), Mars (1989) e Rodrigues (1997) foram, primeiramente, identificadas todas as categorias do sistema e descritos todos os comportamentos que se pretendem estudar, esclarecendo dúvidas nas diferenças de interpretação que existissem. Seguidamente foi discutido o protocolo de observação, treinando qual a codificação mais adequada para os variados comportamentos observados. Para finalizar esta etapa os observadores foram avaliados por um supervisor com o objetivo de verificar se todos eles conheciam bem a definição das variáveis de todo o sistema de observação e realizouse, posteriormente, um período de prática e aplicação do sistema de observação existente.

Fase 2: Desenvolvimento de um novo sistema de observação para o contexto das AGF

Nesta fase o objetivo foi desenvolver as definições do novo sistema de observação ao contexto de AGF verificando se existiam critérios/dimensões e categorias que até então não eram contempladas ao nível dos comportamentos do instrutor no clima de aula, garantindo que o instrumento permita observar o 


\section{Dias, I., Franco, S., Ramos, L., Simões, V.}

que é suposto observar, tendo como referência o sistema de observação de Sarmento et al. (1998). A fim de respeitar a validade de conteúdo, assim como o grau de cobertura do instrumento, segundo Alexandre e Coluci (2011), foi criado um painel de cinco especialistas (painel 1) para que fosse realizado o desenvolvimento do novo sistema recorrendo à literatura existente e à observação de várias aulas de grupo de fitness. Este painel foi integrado por um grupo de sujeitos ligados à intervenção e formação profissional na área das AGF e que possuíssem também competências na área da investigação na intervenção pedagógica em AGF e no desporto em geral, assim como no desenvolvimento e utilização de sistemas de observação. O referido painel efetuou uma análise da bibliografia encontrada relativamente à utilização de critérios/dimensões e categorias de análise do clima de aula nos vários contextos (ensino, treino e Educação Física). Posteriormente, para o desenvolvimento do novo sistema de observação, o painel realizou 3 observações piloto de diferentes AGF, tal como sugerem Anguera e Hernández-Mendo (2013) com diferentes tipos de população (crianças, adultos e idosos), seguidas de discussões que resultaram em várias alterações relativamente ao sistema de observação original. Foi verificado, também pelos experts, como sugerido por Anguera e Hernández-Mendo (2013), a exaustividade e a exclusividade mútua do sistema de observação, garantindo que qualquer comportamento pode ser atribuído a uma das categorias (exaustividade) e que não existe sobreposição entre as categorias incluídas no sistema (mútua exclusividade).

Fase 3: Validação Facial do novo Sistema de Observação por Especialistas (Experts)

Baseado em Brewer e Jones (2002) e Prudente, Garganta e Anguera (2004) foi constituído um segundo painel de especialistas, que nunca tivessem estado envolvidos neste processo anteriormente com o objetivo de avaliar a possibilidade do SOCA-AGF codificar os comportamentos de clima de aula dos instrutores de fitness, em aulas de grupo e verificar se as dimensões e categorias teriam sido devidamente definidas e se seriam relevantes para o referido contexto. Com base nos comentários e sugestões realizadas por este painel de especialistas, foram efetuadas algumas revisões, das quais resultou a versão final do SOCA-AGF.
Fase 4: Fiabilidade Interobservadores em relação ao novo sistema de observação SOCA-AGF

Após concluída a versão final do SOCA-AGF, Brewer e Jones (2002), Gilbert et al. (1999) e Prudente et al. (2004), sugerem a realização da fiabilidade interobservadores, testando desta forma a consistência das observações do novo sistema de observação. Depois de realizada a identificação das categorias do sistema, discutido o protocolo de observação e avaliada a aprendizagem das categorias, pelos dois observadores, foi visionado um vídeo de uma aula de grupo e realizada a respetiva codificação por ambos os observadores, separadamente, não havendo acesso oral ou visual entre eles. Realizada a codificação foi testada a fiabilidade interobservadores a fim de verificar a existência de concordância entre observadores, através da medida de concordância Kappa de Cohen indicada por Pestana e Gageiro (2005). Foram aceites valores de fiabilidade iguais ou superiores a $80 \%$.

\section{Fase 5: Fiabilidade Intraobservadores em relação ao} novo Sistema de Observação

A fidelidade intraobservador tem como objetivo assegurar que existe estabilidade temporal por teste/reteste relativamente ao novo instrumento e ao observador deste estudo, tal como recomendado por Brewer e Jones (2002). O intervalo adotado foi de uma semana entre cada observação. Para este procedimento foi utilizada a medida de concordância Kappa de Cohen indicada por Pestana e Gageiro (2005). Foram aceites valores de fiabilidade iguais ou superiores a $80 \%$.

\section{Aplicação Piloto do SOCA-AGF}

Depois de desenvolvido o SOCA-AGF, foi efetuada a sua aplicação piloto tal como sugerem estudos onde foram desenvolvidos e validados sistemas de observação (Santos, Fernandez, Oliveira, Leitão, Anguera \& Campaniço, 2009; Franco, 2009; Alves et al., 2013; Alves et al., 2014; Alves et al., 2015; Franco \& Simões, 2017). Foram observados e codificados os comportamentos relacionados com o clima de aula de 3 instrutoras da atividade de hidroginástica e 3 instrutoras da atividade de localizada, perfazendo um total de 6 sessões observadas e analisadas para população idosa.

Análise estatística 


\section{Desenvolvimento e Validação do SOCA-AGF - Aplicação Piloto em Idosos}

Relativamente ao desenvolvimento do SOCA-AGF, foi utilizado o teste Kappa de Cohen, para testar as fiabilidades interobservadores e intraobservador para cada uma das categorias do clima de aula que constituem o respetivo sistema. Em relação ao estudo piloto (aplicação do SOCA-AGF), em cada uma das atividades (hidroginástica e localizada), foi contabilizado o registo de frequência de comportamentos por instrutora, assim como o registo de duração dos comportamentos, a duração de cada aula e o registo de ocorrência ("realiza"/"não realiza"), em cada categoria respetiva. Foi determinada a frequência dos comportamentos das instrutoras relativamente ao clima de aula, por minuto, dividindo o número total de ocorrências, pela duração total da sessão em minutos. Para cada categoria do clima de aula e em cada uma das atividades (hidroginástica e localizada), foram calculadas a frequência das categorias de SOCA-AGF (\%) para as categorias cujo registo é de frequências e a duração das categorias de SOCA-AGF (\%), para as categorias cujo registo é de duração de cada instrutora por atividade, bem como a média e desvio padrão de frequência e duração de cada comportamento de clima de aula (\%) nas várias categorias e/ou subcategorias das respetivas dimensões do SOCA-AGF. Para tal, os valores das frequências de comportamento e duração de comportamentos foram depois apresentados em termos percentuais. Toda a análise estatística referida anteriormente foi realizada através do Statistical Package for the Social Sciences (SPSS), versão 20.0. Para a Análise da Generalização foi utilizado o programa SAGT (Hernández-Mendo, BlancoVillaseñor, Pastrana, Morales-Sánchez y RamosPérez, 2016). A Teoria da Generalização (TG) (Cardinet, Tourneur \& Allal, 1976, 1981) unificou as definições de confiabilidade, validade e precisão (Blanco-Villaseñor, Castellano, Hernández-Mendo, Sánchez-López \& Usabiaga, 2014) e é basicamente composta por quatro fases: (1) Definição das facetas de estudo; (2) Análise da variação dos escores obtidos nas facetas do estudo; (3) Cálculo de componentes de erro;(4) Otimização dos coeficientes de Generalização (Blanco-Villaseñor, Castellano, Hernández-Mendo, Sánchez-López \& Usabiaga, 2014).

\section{RESULTADOS}

No que diz respeito à versão final do sistema de observação SOCA-AGF (tabela 1) este apresenta 2 critérios/dimensões, 36 categorias e 8 subcategorias. É apresentada, na tabela 1, também, a seguir ao nome da categoria, o método de registo da mesma, variando entre frequência (F), duração (D) ou se executa ou não recorrendo aos termos Sim e Não (S/N).

No que diz respeito ao processo de desenvolvimento e validação do sistema de observação SOCA-AGF verificou-se que, na fase 4 , relativa à fiabilidade interobservadores (tabela 2), os valores de Kappa de Cohen obtidos foram sempre superiores a $80 \%$, variando entre $96.4 \%$ e $100 \%$. Assim sendo, estes resultados indicam que as definições das categorias do SOCAAGF aparentam ser claras, objetivas e por isso com fiabilidade. $\mathrm{Na}$ fase 5 , relativa à fiabilidade intraobservadores (tabela 2), os valores de Kappa de Cohen variaram entre $86.7 \%$ e $100 \%$. Estes resultados indicam a existência de estabilidade temporal nas observações, refletindo entendimento e compreensão das dimensões e categorias que compõem este novo sistema de observação (SOCA-AGF). Para a análise da generalização foram seguidas as recomendações de Blanco-Villaseñor, Castellano, Hernández-Mendo, Sánchez-López e Usabiaga (2014). Na determinação do número de sessões necessárias para generalizar com precisão realizou-se um desenho baseado em 2 facetas (Categorias e Sessões Observadas $=\mathrm{C} / \mathrm{S}$ ). A estimação dos componentes de variância foi realizada de forma aleatória infinita. A análise mostra uma grande variedade associada à faceta Categorias (64.6\%), seguidas da faceta de interação entre Categorias e Sessões Observadas (33.4\%) e o restante para a faceta Sessões Observadas (2\%). Considerando os índices de generalização para esta estrutura verifica-se (tabela 3) que as 6 sessões observadas no presente estudo permitem um coeficiente de generalização de 0.921 (valor relativo) e 0.916 (valor absoluto). Apesar destes valores serem aceitáveis para um índice de generalização quase perfeito (perto da unidade) seriam necessárias observar 55 sessões, tal como exposto na tabela 3. Os resultados obtidos através do SOCA-AGF referem-se à codificação de um total de 2375 comportamentos de clima de aula de 6 instrutoras em 2 atividades. A duração das aulas de hidroginástica variou entre 37' 40 ' ' e 54'15' enquanto que a localizada variou entre 41'14' e 43'37'. Observou-se que o número de comportamentos de frequência por parte das instrutoras foi sempre superior no critério/dimensão clima positivo (CP) do 


\section{Dias, I., Franco, S., Ramos, L., Simões, V.}

que no critério/dimensão clima negativo $(\mathrm{CN})$ quer seja na atividade de hidroginástica (CP -695.308 e 478 ocorrências; $\mathrm{CN}-0$ e 0 ocorrências), quer seja na atividade de localizada (CP - 383, 356 e 154 ocorrências; $\mathrm{CN}-1$ e 0 ocorrências). Em relação aos comportamentos com registo de duração o mesmo se verificou, ou seja, os comportamentos pertencentes a CP tiveram uma duração sempre superior aos comportamentos de $\mathrm{CN}$ quer na hidroginástica $(\mathrm{CP}-$ 00:23:19, 00:23:08 e 00:15:02; CN - 00:00:00, 00:00:00 e 00:00:00), quer na localizada (CP 00:21:47, 00:16:03 e 00:18:42; CN - 00:00:47, 00:00:00 e 00:00:00). No que diz respeito à frequência de comportamentos por minuto podemos observar que, relativamente a $\mathrm{CP}$, na hidroginástica as instrutoras realizaram maior número de comportamentos por minuto $(\mathrm{M} \pm \mathrm{DP}=11.0 \pm 2.4)$, do que na localizada $(\mathrm{M} \pm \mathrm{DP}=7.0 \pm 3.0)$. No caso do $\mathrm{CN}$ na hidroginástica não existiram casos de registo de frequência $(\mathrm{M} \pm \mathrm{DP}=0.0 \pm 0.0)$, enquanto na localizada existiram comportamentos de registo por parte de uma das instrutoras $(\mathrm{M} \pm \mathrm{DP}=0.0 \pm 0.1)$. Relativamente aos resultados apresentados pelas categorias Cumprimentar e Despedir, que são registadas de acordo com "realiza"/"não realiza" $(\mathrm{S} / \mathrm{N})$ verificou-se que todas as instrutoras de hidroginástica se despediram dos participantes na aula mas apenas uma os cumprimentou no início da aula. Relativamente à atividade de localizada duas das instrutoras cumprimentaram e despediram-se dos praticantes e uma não realizou nenhum destes comportamentos.

Posteriormente serão apresentados (tabela 4) os resultados referentes à frequência dos dados das categorias cujo método de registo é a frequência dos comportamentos de clima de aula (\%) de cada instrutora (I), bem como a média e desvio padrão dos referidos comportamentos (\%) em cada uma das atividades (hidroginástica e localizada), nas várias categorias e subcategorias das respetivas dimensões do clima de aula. Verificou-se que na dimensão CP os comportamentos mais realizados na hidroginástica foram: sorrir (subcategoria da expressão corporal para $\mathrm{CP})(\mathrm{M} \pm \mathrm{DP}=21.2 \pm 1.3 \%)$; inflexão de voz para $\mathrm{CP}(\mathrm{o}$ instrutor muda a entoação de voz, dando ênfase ao discurso $)(\mathrm{M} \pm \mathrm{DP}=16.5 \pm 3.6 \%)$ e movimentação no espaço da aula (o instrutor move-se no espaço da aula, mantendo contacto visual com a classe) $(\mathrm{M} \pm \mathrm{DP}=$ $15.9 \pm 8.5 \%)$.

Nas aulas de hidroginástica observadas existiram comportamentos que nunca foram realizados pelas instrutoras, nomeadamente: contacto físico, elogiar e piscar o olho (subcategoria da expressão corporal para $\mathrm{CP})$. No que diz respeito à dimensão $\mathrm{CN}$ verificou-se que nenhuma das instrutoras realizou qualquer comportamento desta natureza. Na dimensão $\mathrm{CP}$ nas aulas de localizada verificou-se que os comportamentos mais realizados pelas instrutoras de localizada foram: movimentação no espaço da aula $(\mathrm{M} \pm \mathrm{DP}=22.2 \pm 5.0 \%)$, inflexão de voz para $\mathrm{CP}$ $(\mathrm{M} \pm \mathrm{DP}=17.3 \pm 9.8 \%)$ e sorrir (subcategoria da expressão corporal para $\mathrm{CP})(\mathrm{M} \pm \mathrm{DP}=13.6 \pm 4.5 \%)$. Por outro lado, as categorias menos observadas nesta dimensão de análise foram: incluir no exercício $(\mathrm{M} \pm \mathrm{DP}=1.8 \pm 0.6 \%)$; encorajar para a participação $(\mathrm{M} \pm \mathrm{DP}=0.8 \pm 0.5 \%)$ e elogiar $(\mathrm{M} \pm \mathrm{DP}=0.4 \pm 0.3 \%)$. Os comportamentos nunca realizados por nenhuma das instrutoras foram: contacto físico; interação entre praticantes e aceitar as sugestões dos praticantes. Relativamente à dimensão $\mathrm{CN}$ apenas a instrutora I1 realizou comportamentos desta natureza, nomeadamente excluir do exercício (0.3\%). Seguidamente, na tabela 5, serão apresentados os resultados cujo registo foi feito em termos de duração, nomeadamente, para $\mathrm{CP}$, a interação entre participantes, o exercício participativo e o exercício e para $\mathrm{CN}$ o exercício independente e abandonar o espaço da aula. Como se pode constatar através da tabela 5 na atividade de hidroginástica a única categoria com registo de duração foi exercício $(\mathrm{M} \pm \mathrm{DP}=46.9 \pm 13.3 \%)$, que pertence à dimensão $\mathrm{CP}$. A instrutora que mais tempo realizou exercício, segundo os registos foi a instrutora I2 (61.7\%), seguindo-se a instrutora I1 (42.8\%) e por fim a instrutora I3 (36.1\%). Relativamente à dimensão CN, não se verificou qualquer registo de ocorrência. $\mathrm{Na}$ atividade de localizada a categoria com maior duração realizada pelas instrutoras foi exercício $(\mathrm{M} \pm \mathrm{DP}=$ $41.2 \pm 20.9 \%$ ) seguida de exercício participativo $(\mathrm{M} \pm \mathrm{DP}=1.8 \pm 3.2 \%)$. Na categoria exercício, em que o instrutor executa total ou parcialmente o mesmo exercício que os praticantes, sem assumir o papel de praticante, a instrutora que mais tempo realizou exercício, segundo os registos foi a instrutora I1 (43.1\%), seguindo-se a instrutora I3 (41.5\%) e por fim a instrutora I2 (39.0\%). Ao contrário do que se verificou na atividade de hidroginástica, na localizada existiram registos de duração da dimensão $\mathrm{CN}$ por parte da instrutora I1 relativamente à categoria abandonar o espaço da aula (1.1\%). 


\section{Desenvolvimento e Validação do SOCA-AGF - Aplicação Piloto em Idosos}

Tabela 1. Sistema de Observação do Clima de Aula em Aulas de Grupo de Fitness (SOCA-AGF).

\begin{tabular}{|c|c|c|}
\hline \multicolumn{2}{|l|}{ Critério/Dimensão: Clima Positivo } & \multirow{2}{*}{$\begin{array}{l}\text { Critério/Dimensão: Clima Negativo } \\
\text { Categorias }\end{array}$} \\
\hline Categorias & Subcategorias & \\
\hline Cumprimentar - S/N & & Exercício Independente $-\mathrm{D}$ \\
\hline Despedir - S/N & & Abandonar o Espaço da Aula - D \\
\hline \multirow[t]{5}{*}{ Contacto Físico Positivo - F } & Aperto de Mão & Não aceitar as Sugestões do(s) Praticante(s)-F \\
\hline & Acarinhar & Ignorar as Intervenções do(s) Praticante(s) - F \\
\hline & Abraçar & Indiferença/Afastamento $-\mathrm{F}$ \\
\hline & Beijar & Avaliar Negativamente a Participação do \\
\hline & & Praticante no Exercício - F \\
\hline Interação entre Praticantes - D & & Desagrado/Repreensão/Crítica - F \\
\hline \multirow[t]{5}{*}{ Expressão Corporal Positiva - F } & Rir & Ironizar $-\mathrm{F}$ \\
\hline & Sorrir & Ameaçar - F \\
\hline & Piscar o olho & Pressionar para o Exercício - F \\
\hline & Gestos e Expressões & Castigar com Exercício - F \\
\hline & $\begin{array}{l}\text { Corporais Enfáticas } \\
\text { para Clima Positivo }\end{array}$ & \\
\hline Gracejar - F & & Excluir do Exercício - F \\
\hline Elogiar - F & & Expulsar da Aula - F \\
\hline Conversar com os Praticantes-F & & Agressão Física - F \\
\hline Questionar - F & & Inflexão de Voz para Clima Negativo - F \\
\hline Identificar o Praticante $-\mathrm{F}$ & & Expressão Corporal para Clima Negativo - F \\
\hline Atenção às intervenções dos & & \\
\hline Praticantes $-\mathrm{F}$ & & \\
\hline Aceitar as intervenções dos & & \\
\hline Praticantes $-\mathrm{F}$ & & \\
\hline Avaliar Positivamente a & & \\
\hline participação do Praticante no & & \\
\hline Exercício - F & & \\
\hline Encorajar para a Participação- F & & \\
\hline Emissão de Sons - F & & \\
\hline Incluir no Exercício - F & & \\
\hline Exercício Participativo - D & & \\
\hline Exercício - D & & \\
\hline Inflexão de Voz para Clima & & \\
\hline Positivo $-\mathrm{F}$ & & \\
\hline Movimentação no Espaço da & & \\
\hline Aula - F & & \\
\hline
\end{tabular}




\section{Dias, I., Franco, S., Ramos, L., Simões, V.}

Tabela 2. Fiabilidade interobservador e intraobservador do instrumento SOCA-AGF.

\begin{tabular}{|c|c|c|c|c|c|}
\hline & \multicolumn{3}{|c|}{ Critério/Dimensão: Clima Positivo } & \multicolumn{2}{|c|}{ Critério/Dimensão: Clima Negativc } \\
\hline Categorias & & $\begin{array}{l}\text { Kappa } \\
\text { Cohen } \\
\text { Interobse } \\
\text { rvador }\end{array}$ & $\begin{array}{l}\text { Kappa de } \\
\text { Cohen } \\
\text { Intraobser } \\
\text { vador }\end{array}$ & Categorias & $\begin{array}{l}\text { Kappa } \\
\text { Cohen } \\
\text { Interol } \\
\text { rvador }\end{array}$ \\
\hline & Subcategorias & & & & \\
\hline Cumprimentar & & \# & \# & Exercício Independente & \# \\
\hline Despedir & & \# & \# & $\begin{array}{l}\text { Abandonar o Espaço da } \\
\text { Aula }\end{array}$ & $\#$ \\
\hline \multirow[t]{4}{*}{$\begin{array}{l}\text { Contacto Físico } \\
\text { Positivo }\end{array}$} & Aperto de Mão & \# & \# & $\begin{array}{l}\text { Não aceitar as Sugestões } \\
\text { do(s) Praticante(s) }\end{array}$ & \# \\
\hline & Acarinhar & \# & \# & $\begin{array}{l}\text { Ignorar as Intervenções } \\
\text { do(s) Praticante(s) }\end{array}$ & \# \\
\hline & Abraçar & \# & \# & Indiferença/Afastamento & \# \\
\hline & Beijar & \# & \# & $\begin{array}{l}\text { Avaliar Negativamente a } \\
\text { Participação do } \\
\text { Praticante no Exercício }\end{array}$ & \# \\
\hline $\begin{array}{l}\text { Interação entre } \\
\text { Praticantes }\end{array}$ & & \# & \# & $\begin{array}{l}\text { Desagrado/Repreensão/ } \\
\text { Crítica }\end{array}$ & $1.000 *$ \\
\hline Expressão Corporal & Rir & $1.000 *$ & $0.929 *$ & Ironizar & \# \\
\hline \multirow[t]{3}{*}{ Positiva } & Sorrir & $0.968 *$ & $0.938 *$ & Ameaçar & \# \\
\hline & Piscar o olho & \# & \# & $\begin{array}{l}\text { Pressionar para o } \\
\text { Exercício }\end{array}$ & \# \\
\hline & $\begin{array}{l}\text { Gestos e } \\
\text { Expressões } \\
\text { Corporais } \\
\text { Enfáticas para } \\
\text { Clima Positivo }\end{array}$ & $1.000 *$ & $1.000 *$ & Castigar com Exercício & \# \\
\hline Gracejar & & $0.973 *$ & $0.972 *$ & Excluir do Exercício & \# \\
\hline Elogiar & & \# & \# & Expulsar da Aula & \# \\
\hline $\begin{array}{l}\text { Conversar com os } \\
\text { Praticantes }\end{array}$ & & $1.000 *$ & $0.867 *$ & Agressão Física & \# \\
\hline Questionar & & $1.000 *$ & $0.919 *$ & $\begin{array}{l}\text { Inflexão de Voz para } \\
\text { Clima Negativo }\end{array}$ & \# \\
\hline Identificar o Praticante & & $1.000 *$ & $0.937 *$ & $\begin{array}{l}\text { Expressão Corporal para } \\
\text { Clima Negativo }\end{array}$ & $\#$ \\
\hline $\begin{array}{l}\text { Atenção às intervenções } \\
\text { dos Praticantes }\end{array}$ & & $0.975^{*}$ & $0.975^{*}$ & & \\
\hline $\begin{array}{l}\text { Aceitar as intervenções } \\
\text { dos Praticantes }\end{array}$ & & \# & \# & & \\
\hline $\begin{array}{l}\text { Avaliar Positivamente } \\
\text { a participação do } \\
\text { Praticante no Exercício }\end{array}$ & & $1.000 *$ & $0.929 *$ & & \\
\hline $\begin{array}{l}\text { Encorajar para a } \\
\text { Participação }\end{array}$ & & \# & \# & & \\
\hline Emissão de Sons & & \# & \# & & \\
\hline Incluir no Exercício & & $1.000 *$ & $0.905^{*}$ & & \\
\hline Exercício Participativo & & \# & \# & & \\
\hline Exercício & & $0.964 *$ & $0.938 *$ & & \\
\hline $\begin{array}{l}\text { Inflexão de Voz para } \\
\text { Clima Positivo }\end{array}$ & & $1.000 *$ & $0.937 *$ & & \\
\hline $\begin{array}{l}\text { Movimentação no } \\
\text { Espaço da Aula }\end{array}$ & & $1.000 *$ & $1.000 *$ & & \\
\hline
\end{tabular}




\section{Desenvolvimento e Validação do SOCA-AGF - Aplicação Piloto em Idosos}

Tabela 3. Estudo de decisão da análise de generalização para estimar o número de sessões.

\begin{tabular}{llll}
\hline & 6 sessões & 35 sessões & 55 sessões \\
\hline Índice de fiabilidade (coef G relativo) & 0.921 & 0.985 & 0.991 \\
Índice de generalidade (coef G absoluto) & 0.916 & 0.986 & 0.990 \\
\hline
\end{tabular}

\section{DISCUSSÃO}

Para desenvolver e validar o SOCA-AGF foram seguidos os pressupostos recomendados por diversos autores e utilizados para a validação de instrumentos similares (Cardinet, Tourneur \& Allal, 1976, 1981; Gilbert, Trudel, Gaumond \& Larocque, 1999; Anguera, Blanco, Losada \& Hernández-Mendo, 2000; Brewer \& Jones, 2002; Prudente, Garganta \& Anguera, 2004, Hernández-Mendo, Martínez \& Moráles-Sánchez, 2010; Costa, Garganta, Greco, Mesquita \& Maia, 2011; Franco, 2009; HernándezMendo, Ramos-Pérez \& Pastrana, 2012; Alves et al., 2013; Alves et al., 2014; Blanco-Villaseñor, Castellano, Hernández-Mendo, Sánchez-López \& Usabiaga, 2014; Franco \& Simões, 2017). A fiabilidade interobservador e intraobservador apresentou resultados que indicaram que as definições das categorias do SOCA-AGF aparentam ser claras, objetivas e com fiabilidade. A análise dos coeficientes de generalidade, nesta estrutura de desenho, revela uma elevada fiabilidade de precisão de generalização. Posteriormente ao desenvolvimento e validação do SOCA-AGF, foi testada a funcionalidade do instrumento, através de uma aplicação piloto, numa amostra de 6 instrutoras de 2 atividades (hidroginástica e localizada), em população idosa. Foram codificados um total de 2375 comportamentos que influenciam o clima de aula nas 6 aulas analisadas, o que resulta numa média de 396 comportamentos por aula.

Assim sendo, o SOCA-AGF aparenta ter um carácter inovador neste contexto de intervenção, mostrando-se não só ser uma ferramenta eficaz para a codificação dos comportamentos realizados pelos instrutores de fitness, nos diferentes tipos de aulas de grupo, como também na obtenção de dados de forma casuística e multidimensional.

Ao ser realizada uma leitura mais pormenorizada na análise de cada critério/dimensão e respetivas categorias por atividade, verificou-se que no $\mathrm{CP}$ o exercício foi a categoria mais realizada pelas instrutoras nas atividades de hidroginástica e localizada (46.9\% e $41.2 \%$, respetivamente). Estes valores podem ser resultado da opção das instrutoras em realizar simultaneamente o exercício com os praticantes servindo de modelo/referência, facilitando a aprendizagem e motivando os praticantes. Poderá também estar relacionado com o estilo de ensino utilizado pelas instrutoras, designadamente $o$ frequentemente utilizado neste tipo de aulas, o estilo de ensino por comando, onde o instrutor toma todas as decisões acerca dos exercícios, enquanto os participantes seguem os exercícios do instrutor (Francis \& Seibert, 2000). Por sua vez, ainda na dimensão $\mathrm{CP}$ as categorias nunca realizadas foram: interação entre praticantes $(0.0 \%)$ e contacto físico positivo, assim como as subcategorias que dele fazem parte. Tal como Franco e Simões (2017) constataram, num estudo realizado ao nível da emissão de feedbacks, na atividade de hidroginástica, as instrutoras nunca utilizaram o contacto físico ou a manipulação corporal, possivelmente pelo facto dos praticantes se encontrarem dentro de água e o instrutor estar no cais da piscina. Relativamente à categoria expressão corporal para $\mathrm{CP}$, que consiste em o instrutor utilizar a sua expressão corporal com o objetivo de criar um clima de aula positivo, da qual fazem parte as subcategorias: rir, sorrir, piscar o olho e gestos e expressões corporais enfáticas para clima positivo, este teve em média as percentagens mais elevadas nas aulas de hidroginástica $(5.2 \%, 21.3 \%$, $0.0 \%$ e $13.7 \%$, respetivamente) do que nas de localizada $(5.0 \%, 13.6 \%, \quad 0.0 \%$ e $11.9 \%$, respetivamente). Frequente é a categoria gracejar com uma média de percentagens de $10.7 \%$ nas aulas de hidroginástica e de $13.2 \%$ nas aulas de localizada. Para Loughead, Colman e Carron (2001), é bastante propício que este tipo de comportamento predomine face à importância que os mesmos podem ter no entusiasmo e adesão dos praticantes. A categoria conversar com os praticantes apresenta valores 


\section{Dias, I., Franco, S., Ramos, L., Simões, V.}

bastante baixos quer nas aulas de hidroginástica $(1.2 \%)$, quer nas aulas de localizada (2.3\%). Importa referir que dois dos momentos mais propícios para conversar com os praticantes, sugerida inclusive como estratégia para aumentar a adesão (Carron, Hausenblas \& Estabrooks, 1999), são antes e depois da aula propriamente dita (Brehm, 2004). Possivelmente os resultados da categoria conversar com os praticantes aparecem com valores baixos pelo facto desses momentos não terem sido filmados na observação com o objetivo de estandardizar o início e final da aula e até por questões éticas, face à variabilidade existente nestes momentos $\mathrm{O}$ questionar mostrando preocupação com o praticante acerca do exercício encontra-se presente como comportamento realizado pelas instrutoras, ainda que apresente uma média relativamente baixa (hidroginástica $2.2 \%$, localizada $5.6 \%$ ). Quanto à categoria de atenção às intervenções dos praticantes (hidroginástica 2.1\%, localizada 5\%), esta apresenta valores da média ligeiramente inferiores relativamente à categoria questionar. Dada a importância que essa demonstração de interesse possa ter no clima relacional (Sarmento et al., 1998), perspetiva-se que os baixos valores médios obtidos nesta categoria não derivem do facto do instrutor não prestar atenção às intervenções dos praticantes, mas sim pelas características próprias da atividade, onde é utilizada música, dificultando a receção da informação auditiva e consequentemente a comunicação entre os intervenientes. As instrutoras optaram por não corrigir os alunos muitas vezes o que pode ter ocorrido pelo facto de terem existido poucos erros ou evitar evidenciar que os praticantes erram e assim estes se sentirem mais competentes na realização dos exercícios, o que contribui para a satisfação e adesão dos praticantes. Sarmento et al. (1998: 4-5) acrescenta que:"(...) o julgamento excessivo dos comportamentos observados pode dificultar um bom clima relacional.". Carron, Hausenblas e Estabrooks (1999) referem ainda que "ignorar os enganos" poderá constituir uma estratégia para aumentar a autoeficácia e a adesão. Estas referências vão ao encontro dos resultados obtidos no presente estudo em que a categoria avaliar positivamente a participação do praticante no exercício possui uma média percentual de $5.4 \%$ nas aulas de hidroginástica e $2.3 \%$ nas aulas de localizada. Os instrutores, em média, elogiam e avaliam positivamente mais do que denigrem a prestação dos praticantes. Segundo Franco (2009), é importante que o instrutor, ao exprimir-se, utilize o canal de comunicação mais adequado tendo por isso sempre em conta a situação, o contexto e o espaço físico onde se desenrola a atividade. No presente estudo verificou-se que as categorias emissão de sons e a inflexão de voz para clima positivo, foram mais utilizadas nas aulas de localizada ( $7.8 \%$ e $17.3 \%$ respetivamente) do que nas aulas de hidroginástica $(3.3 \%$ e $16.5 \%$ respetivamente). Este acontecimento poderá ser justificado por, em ambas as atividades, haver utilização de música. O som que advém da música pode dificultar a receção da informação auditiva por parte dos praticantes, particularmente nas aulas de hidroginástica em que as condições acústicas são, geralmente, menos boas e a comunicação visual assume particular importância. Possivelmente por estas razões e tal como nos mostram os resultados obtidos neste estudo, a subcategoria gestos e expressões corporais enfáticas positivas possui uma média percentual de $13.7 \%$ nas aulas de hidroginástica e de $11.9 \%$ nas aulas de localizada.

Tal como afirma Castañer, Camerino, Anguera e Jonsson (2010), para que os instrutores sejam eficazes na sua instrução, é fundamental que estes possuam competências ótimas nos domínios da comunicação não-verbal, nomeadamente ao nível da proxémia com relação aos seus praticantes. No caso da categoria movimentação no espaço da aula, as percentagens de frequência foram de $15.9 \%$ na hidroginástica e de $22.2 \%$ na localizada. Tal como Franco (2009) concluiu, as posições e características dos exercícios podem influenciar os comportamentos dos instrutores. Talvez porque nas atividades de hidroginástica e localizada a execução dos exercícios é realizada sob um conjunto de séries e repetições, muitas vezes é comum os instrutores demonstrarem nas primeiras repetições os exercícios e depois "libertam-se" para poderem fazer movimentações no espaço da aula, de forma a observar a execução dos praticantes, diagnosticar e controlar o que está a acontecer na aula. No critério/dimensão $\mathrm{CP}$, relativamente à hidroginástica, o que se verificou foi que não existiram comportamentos observáveis em nenhuma das categorias (comportamentos de frequência ou de duração) por parte de nenhuma das instrutoras (I1, I2 e I3). 


\section{Desenvolvimento e Validação do SOCA-AGF - Aplicação Piloto em Idosos}

Tabela 4. Frequência das categorias do sistema de observação SOCA-AGF de cada uma das instrutoras

\begin{tabular}{|c|c|c|c|c|c|c|c|}
\hline Categorias/Subcategorias & $\begin{array}{l}\text { I1 } \\
\text { Hidro }\end{array}$ & $\begin{array}{l}\text { I2 } \\
\text { Hidro }\end{array}$ & $\begin{array}{l}\text { I3 } \\
\text { Hidro }\end{array}$ & $\begin{array}{l}\mathrm{M} \pm \mathrm{DP} \\
\text { Hidro }\end{array}$ & $\begin{array}{c}\text { I1 } \\
\text { Local }\end{array}$ & $\begin{array}{c}\mathrm{I} 2 \\
\text { Local }\end{array}$ & $\begin{array}{c}\text { I3 } \\
\text { Local }\end{array}$ \\
\hline \multicolumn{8}{|c|}{ Clima de Aula Positivo } \\
\hline Subcategorias Aperto de Mão & 0.0 & 0.0 & 0.0 & $0.0 \pm 0.0$ & 0.0 & 0.0 & 0.0 \\
\hline do Contacto & 0.0 & 0.0 & 0.0 & $0.0 \pm 0.0$ & 0.0 & 0.0 & 0.0 \\
\hline Abraçar & 0.0 & 0.0 & 0.0 & $0.0 \pm 0.0$ & 0.0 & 0.0 & 0.0 \\
\hline Positivo & 0.0 & 0.0 & 0.0 & $0.0 \pm 0.0$ & 0.0 & 0.0 & 0.0 \\
\hline Rir & 10.5 & 3.6 & 1.7 & $5.2 \pm 4.6$ & 3.1 & 5.5 & 6.5 \\
\hline Sorrir & 22.7 & 20.7 & 20.3 & $\begin{array}{c}21.2 \pm 1.3 \\
0.0+00\end{array}$ & 10.4 & 18.8 & 11.7 \\
\hline $\begin{array}{cl}\text { da Expressão } & \text { Gestos e } \\
\text { Corporal do } & \text { Expressões }\end{array}$ & & & & & & & \\
\hline $\begin{array}{cl}\text { Clima } & \text { Corporais } \\
\text { Positivo } & \text { Enfáticas para } \\
& \text { Clima Positivo }\end{array}$ & 11.9 & 11.3 & 17.7 & $13.7 \pm 3.5$ & 10.2 & 19.1 & 6.5 \\
\hline Gracejar & 20.1 & 6.1 & 5.8 & $10.7 \pm 8.1$ & 16.7 & 23.0 & 0.0 \\
\hline Elogiar & 0.0 & 0.0 & 0.0 & $0.0 \pm 0.0$ & 0.5 & 0.0 & 0.6 \\
\hline Conversar com os Praticantes & 1.1 & 2.3 & 0.2 & $1.2 \pm 1.0$ & 1.6 & 3.9 & 1.3 \\
\hline Questionar & 1.4 & 5.2 & 0.0 & $2.2 \pm 2.7$ & 7.3 & 3.1 & 6.5 \\
\hline Identificar o Praticante & 2.0 & 1.9 & 0.2 & $1.4 \pm 1.0$ & 4.2 & 1.2 & 5.8 \\
\hline $\begin{array}{l}\text { Atenção às Intervenções dos } \\
\text { Praticantes }\end{array}$ & 2.9 & 2.3 & 1.3 & $2.1 \pm 0.8$ & 6.3 & 7.4 & 1.3 \\
\hline Aceitar as Sugestões dos Praticantes & 0.0 & 0.3 & 0.0 & $0.1 \pm 0.2$ & 0.0 & 0.0 & 0.0 \\
\hline Avaliar Positivamente a & & & & & & & \\
\hline $\begin{array}{l}\text { Participação do Praticante no } \\
\text { Exercício }\end{array}$ & 2.4 & 1.6 & 12.1 & $5.4 \pm 5.8$ & 1.8 & 2.3 & 2.6 \\
\hline Encorajar para a Participação & 0.3 & 0.0 & 0.4 & $0.2 \pm 0.2$ & 1.3 & 0.4 & 0.6 \\
\hline Emissão de Sons & 1.4 & 6.1 & 2.3 & $3.3 \pm 2.5$ & 0.5 & 5.5 & 17.5 \\
\hline Incluir no Exercício & 1.1 & 0.6 & 0.0 & $0.6 \pm 0.6$ & 2.3 & 1.2 & 1.9 \\
\hline Inflexão de Voz para Clima Positivo & 14.9 & 13.9 & 20.7 & $16.5 \pm 3.6$ & 14.6 & 28.1 & 9.1 \\
\hline Movimentação no Espaço da Aula & 6.9 & 23.6 & 17.1 & $15.9 \pm 8.5$ & 19.0 & 19.5 & 27.9 \\
\hline \multicolumn{8}{|c|}{ Clima de Aula Negativo } \\
\hline $\begin{array}{l}\text { Não Aceitar as Sugestões do(s) } \\
\text { Praticante(s) }\end{array}$ & 0.0 & 0.0 & 0.0 & $0.0 \pm 0.0$ & 0.0 & 0.0 & 0.0 \\
\hline 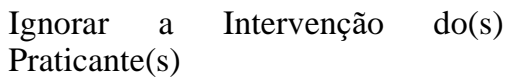 & 0.0 & 0.0 & 0.0 & $0.0 \pm 0.0$ & 0.0 & 0.0 & 0.0 \\
\hline Indiferença/Afastamento & 0.0 & 0.0 & 0.0 & $0.0 \pm 0.0$ & 0.0 & 0.0 & 0.0 \\
\hline Avaliar Negativamente & & & & & & & \\
\hline $\begin{array}{l}\text { Participação do Praticante no } \\
\text { Exercício }\end{array}$ & 0.0 & 0.0 & 0.0 & $0.0 \pm 0.0$ & 0.0 & 0.0 & 0.0 \\
\hline Desagrado/Repreensão/Crítica & 0.0 & 0.0 & 0.0 & $0.0 \pm 0.0$ & 0.0 & 0.0 & 0.0 \\
\hline Ironizar & 0.0 & 0.0 & 0.0 & $0.0 \pm 0.0$ & 0.0 & 0.0 & 0.0 \\
\hline Ameaçar & 0.0 & 0.0 & 0.0 & $0.0 \pm 0.0$ & 0.0 & 0.0 & 0.0 \\
\hline Pressionar para o Exercício & 0.0 & 0.0 & 0.0 & $0.0 \pm 0.0$ & 0.0 & 0.0 & 0.0 \\
\hline Castigar com Exercício & 0.0 & 0.0 & 0.0 & $0.0 \pm 0.0$ & 0.0 & 0.0 & 0.0 \\
\hline Excluir do Exercício & 0.0 & 0.0 & 0.0 & $0.0 \pm 0.0$ & 0.3 & 0.0 & 0.0 \\
\hline Expulsar da Aula & 0.0 & 0.0 & 0.0 & $0.0 \pm 0.0$ & 0.0 & 0.0 & 0.0 \\
\hline Agressão Física & 0.0 & 0.0 & 0.0 & $0.0 \pm 0.0$ & 0.0 & 0.0 & 0.0 \\
\hline $\begin{array}{l}\text { Inflexão de Voz para Clima } \\
\text { Negativo }\end{array}$ & 0.0 & 0.0 & 0.0 & $0.0 \pm 0.0$ & 0.0 & 0.0 & 0.0 \\
\hline $\begin{array}{l}\text { Expressão Corporal para Clima } \\
\text { Negativo }\end{array}$ & 0.0 & 0.0 & 0.0 & $0.0 \pm 0.0$ & 0.0 & 0.0 & 0.0 \\
\hline
\end{tabular}

Instrutor 1 (I1); Instrutor 2 (I2); Instrutor 3 (I3); Média (M); Desvio Padrão (DP); Hidroginástica (Hidro); Localizada (Local). 
Dias, I., Franco, S., Ramos, L., Simões, V.

Tabela 5. Duração das categorias do sistema de observação SOCA-AGF de cada uma das instrutoras

\begin{tabular}{|c|c|c|c|c|c|c|c|c|}
\hline Categorias/Subcategorias & $\begin{array}{c}\text { I1 } \\
\text { Hidro }\end{array}$ & $\begin{array}{c}\text { I2 } \\
\text { Hidro }\end{array}$ & $\begin{array}{c}\text { I3 } \\
\text { Hidro }\end{array}$ & $\begin{array}{c}\mathrm{M} \pm \mathrm{DP} \\
\text { Hidro }\end{array}$ & $\begin{array}{c}\text { I1 } \\
\text { Local }\end{array}$ & $\begin{array}{c}\text { I2 } \\
\text { Local }\end{array}$ & $\begin{array}{c}\text { I3 } \\
\text { Local }\end{array}$ & $\begin{array}{c}\mathrm{M} \pm \mathrm{DP} \\
\text { Local }\end{array}$ \\
\hline \multicolumn{9}{|c|}{ Clima de Aula Positivo } \\
\hline Interação entre participantes & 0.0 & 0.0 & 0.0 & $0.0 \pm 0.0$ & 0.0 & 0.0 & 0.0 & $0.0 \pm 0.0$ \\
\hline Exercício participativo & 0.0 & 0.0 & 0.0 & $0.0 \pm 0.0$ & 5.5 & 0.0 & 0.0 & $1.8 \pm 3.2$ \\
\hline Exercício & 42.8 & 61.7 & 36.1 & $46.9 \pm 13.3$ & 43.1 & 39.0 & 41.5 & $41.2 \pm 20.9$ \\
\hline \multicolumn{9}{|c|}{ Clima de Aula Negativo } \\
\hline Exercício Independente & 0.0 & 0.0 & 0.0 & $0.0 \pm 0.0$ & $\overline{0.0}$ & 0.0 & 0.0 & $0.0 \pm 0.0$ \\
\hline Abandonar o Espaço da Aula & 0.0 & 0.0 & 0.0 & $0.0 \pm 0.0$ & 1.1 & 0.0 & 0.0 & $0.4 \pm 0.1$ \\
\hline
\end{tabular}

Quanto à localizada, ainda dentro da dimensão "clima negativo", o que se constatou é que as instrutoras I2 e I3 não realizaram qualquer comportamento observável pertencente às categorias desta dimensão, por outro lado, a instrutora I1 apresentou comportamentos de frequência inseridos na categoria excluir do exercício $(0.3 \%)$ e comportamentos de duração inseridos na categoria abandonar o espaço de aula (1.1\%).

Os citados resultados são apoiados por Carron, Hausenblas e Estabrooks (1999) quando este afirma que o instrutor ao desenvolver um bom clima assume um papel importante, potencializando uma importante estratégia de intervenção pedagógica que os instrutores podem e devem utilizar a fim de conseguirem a permanência dos praticantes nas suas aulas.

\section{APLICAÇÕES PRÁTICAS}

Com o desenvolvimento deste estudo espera-se ter dado um contributo ao nível do conhecimento científico na área da pedagogia, em particular no que diz respeito ao comportamento observável dos instrutores em aulas de grupo, que influencia o clima de aula, no contexto do fitness. Espera-se também que a existência de um instrumento desta natureza (SOCAAGF) possa constituir-se como uma ferramenta não só para a área da investigação como também para a área de aplicação profissional, permitindo aos investigadores, instrutores de fitness, diretores técnicos de ginásios/piscinas e demais profissionais da área a utilização deste instrumento para autoanálise, supervisão ou recrutamento auxiliando no conhecimento, avaliação, caracterização e comparação dos comportamentos dos instrutores de fitness em aulas de grupo. A utilização deste sistema de observação poderá apoiar os profissionais da área a diagnosticarem, reforçarem ou modificarem os seus comportamentos na sua atuação profissional, utilizando assim estratégias adequadas em benefício dos seus praticantes promovendo, assim, a adesão dos mesmos. Tem existido ao longo dos anos um amplo debate sobre a utilização das metodologias tradicionalmente designadas por quantitativa e qualitativa, dando abertura à realização de investigações que privilegiam a utilização destas duas metodologias em complemento uma da outra ("mixedmethod research"). Exemplos desta questão, no contexto do fitness, são: estudo realizado por Franco, Rodrigues e Castañer (2012), em que os autores analisaram o comportamento de 62 instrutores em aulas de localizada relacionando-o com as preferências e níveis de satisfação dos praticantes; estudo realizado por Franco e Simões (2017), no qual os autores analisaram os comportamentos de feedback de 12 instrutores de fitness em aulas de hidroginástica, indoor cycling, step e localizada bem como o estudo de Anguera, Camerino, Castañer, Sánchez-Algarra e Onwuegbuzie (2017), onde é referida a importância e pertinência da junção destas duas metodologias. Com recurso à utilização do SOCA-AGF e de outros instrumentos e metodologias poderia, por exemplo, ser interessante estudar o comportamento observável dos instrutores de fitness e relacionar esse comportamento com a sua auto perceção, bem como com as preferências dos praticantes, para tal seria interessante desenvolver um questionário com base no SOCAAGF que permita, por um lado, avaliar a auto perceção dos instrutores, por outro, a preferência dos praticantes relativamente aos comportamentos de clima de aula dos instrutores. Espera-se que esta investigação seja também um estímulo para o desenvolvimento de outros instrumentos de observação sobre outros aspetos pedagógicos na área do fitness. 


\section{Desenvolvimento e Validação do SOCA-AGF - Aplicação Piloto em Idosos}

\section{REFERÊNCIAS}

1. Alexandre, N., \& Coluci, M. (2011). Validade de conteúdo nos processos de construção e adaptação de instrumentos de medidas. Ciência \& Saúde Coletiva, 16(7),3061-3068.

https://doi.org/10.1590/S1413812320110008000 06.

2. Alves, S., Franco, S., Castañer. M., Camerino, O., Rodrigues, J., Hileno, R. (2015). Análise da comunicação paraverbal cinésica e proxêmica em instrutores de fitness através de padrões temporais (T-patterns). Cuadernos de Psicología del Deporte,15(1),111-122.

https://doi.org/10.4321/S1578842320150001000 11.

3. Alves, S., Rodrigues, J., Castañer. M., Camerino, O., Sequeira, P., Carvalhinho, L., Simões, V., Franco, S. (2013). Sistema de observação proxémica do instrutor de fitness (SOPROXFITNESS): Desenvolvimento, validação e estudo piloto. Revista Iberoamericana de Psicología del Ejercicio y el Deporte, 8(2), 281-299.

4. Alves. S., Rodrigues, J., Castañer. M., Camerino, O., Sequeira, P., Carvalhinho, L., Simões, V., Franco, S. (2014). Validação e Desenvolvimento de um Sistema de Observação da Comunicação Cinésica do Instrutor de fitness. Motricidade, 10 (1),77-87.

https://doi.org/10.6063/motricidade.10(1).2638.

5. Anguera, M.T., Blanco, Losada \& HernándezMendo, A. (2000). La metodología observacional en el deporte: conceptos básicos. [Electronic Version]. Lecturas: Educación Física y Deportes, 5. Retrieved 25/5/2019 from http://www.efdeportes.com/efd24b/obs.htm.

6. Anguera, M. T. (2003). La observación. En C. Moreno (Ed.). Evaluación psicológica. Concepto, proceso y aplicación en las áreas del desarrollo y de la inteligencia. Madrid: Sanz y Torres.

7. Anguera, M.T., Blanco-Villaseñor, A. (2003). Registro y codificación en el comportamiento deportivo. En A. Hernández-Mendo, A. (Coord.), Psicología del Deporte (Vol. 2). Metodología (p. 6-34). Buenos Aires: Efdeportes (www.efdeportes.com) [Reimpreso en A. Hernández-Mendo (Coord.) (2005). Psicología del Deporte, Vol. II, Metodología (pp. 33-66). Sevilla: Wanceulen].

8. Anguera, M.T., Magnusson, M.S., Jonsson, G.K. (2007). Instrumentos no estándar. Avances en Medición, 5(1), 63-82.

9. Anguera, M.T., Hernández-Mendo, A. (2013). A metodologia observacional no campo do esporte. E-balonmano.com: Journal of Sports Sciences, 9(3),135-160.

http://www.ebalonmano.com/ojs/index.php/revist a/index.

10. Anguera, M.T., Camerino, O., Castañer, M., Sánchez-Algarra, P., Onwuegbuzie, A.J. (2017). The Specificity of Observational Studies in Physical Activity and Sports Sciences: Moving Forward in Mixed Methods Research and Proposals for Achieving Quantitative and Qualitative Symmetry. Frontiers in Psychology, 8(2196). DOI: 10.3389/fpsyg.2017.02196.

11. Bakeman, R., Quera, V., Gnisci, A. (2009). Concordância entre observadores para dados sequenciais de eventos programados: uma comparação de algoritmos baseados em tempo e baseados em eventos. Behav Res. Methods, 41(1), 137-47. https://doi.org/10.3758/BRM.41.1.137.

12. Blanco-Villaseñor, A., Castellano, J., HernándezMendo, A., Sánchez-López, C.R. \& Usabiaga, O. (2014). Aplicacíon de la TG en el deporte para el studio de la fiabilidade, validez y estimación de la muestra. Revista de Psicología del Deporte, 23(1), 131-137. ISSN: 1132-239X. Disponível em: https://www.redalyc.org/articulo.oa?id=2351 $\not 235129571014$

13. Bray, S. R., Gyurcsik, N. C., Culos-Reed, S. N., Dawson, K. A., \& Martin, K. A. (2001). An exploratory investigation of the relationship between proxy efficacy, self-efficacy and exercise attendance. Journal of Health Psychology, 6(4), 425-434.

https://doi.org/10.1177/135910530100600405

14. Brehm, B. A. (2004). Successful fitness motivation strategies. Champaign, II: Human Kinetics.

15. Brewer, B., \& Jones, R. L. (2002). A five-stage process for establishing contextually valid systematic observation instruments: the case of rugby union. The Sport Pshychologist, 16(2), 138159. https://doi.org/10.1123/tsp. 16.2 .138 


\section{Dias, I., Franco, S., Ramos, L., Simões, V.}

16. Cardinet, J. Tourneur, Y. \& Allal, L. (1976). The simmetry of generalizability theory: Applications to educational measurement. Journal of Educational Measurement,13(2),119-135. http://doi.org/10.1111/j.17453984.1976.tb00003. $\mathrm{X}$

17. Cardinet, J. Tourneur, Y. \& Allal, L. (1981). Extension of generalizability theory and its applications in educational measurement. Journal of Educational Measurement,18(4),183-204. http://doi.org/10.1111/j.17453984.1981.tb00852. $\mathrm{x}$

18. Carreiro da Costa, F. (1998). O Sucesso pedagógico em educação física. Estudo das condições e factores de ensino-aprendizagem associados ao êxito numa unidade de ensino. Tese de Doutoramento, Instituto Superior de Educação Física. Universidade Técnica de Lisboa, Cruz Quebrada. Lisboa.

19. Carron, A. V., Hausenblas, H. A., \& Estabrooks, P. A. (1999). Social influence and exercise involvement. Adherence Issues in Sport and Exercise (pp. 1-17). West Sussex: John Wiley \& Sons Ltd.

20. Carron, A. V., Hausenblas, H. A., \& Mack, D. (1996). Social influence and exercise: A metaAnalysis. Journal of Sport and Exercise Psychology, 18, 1-16. https://doi.org/10.1123/jsep.18.1.1

21. Castañer, M., Camerino, O., Anguera, M. T., \& Jonsson, G. K. (2010). Observing the paraverbal communicative style of expert and novice $\mathrm{PE}$ teachers by means SOCOP: a sequential analysis. Procedia - Social and Behavioral Sciences, 2(2), 5162-67. https://doi.org/10.1016/j.sbspro.2010.03.839

22. Chacón-Moscoso, S., Sanduvete-Chaves, S., Anguera, M.T., Losada, J.L., Portell, M., LozanoLozano, J.A. (2018). Preliminary Checklist for Reporting Observational Studies in Sports Areas: Content Validity. Frontiers in Psychology, 9(291). DOI: 10.3389/fpsyg.2018.00291

23. Collishaw, A., Dyer, L., \& Boies, K. (2008). The authenticity of positive emotional displays: client responses to leisure service employees. Journal of Leisure Research, 40(1), 23-46. https://doi.org/10.1080/00222216.2008.11950131
24. Costa, I., Garganta, J., Greco, P., Mesquita, I., \& Maia, J. (2011). Sistema de avaliação táctica no futebol (FUT-SAT): desenvolvimento e validação preliminar. Motricidade, 7(1), 69-84. https://doi.org/10.6063/motricidade.7(1).121

25. European Health \& Fitness Association (EHFA) (2012). European Health \& Fitness Association, Annual Report. Brussels.

26. Franco, S. \& Simões, V. (2017). Fitness professionals pedagogical intervention. European Journal for Exercise Professionals - Europe Active, 1(1), 29-38.

27. Franco, S. (2009). Comportamento pedagógico dos instrutores de fitness em aulas de grupo de localizada. Comportamento observado, percepção, preferência e satisfação dos praticantes. Tese de Douturamento, Universitat de Lleida - Institut Nacional D'Educació Física de Catalunya, Lleida.

28. Franco, S., \& Simões, V. (2006). Participants' perception and preference about body pump ${ }^{\circledR}$ instructors' pedagogical feedback. Paper presented at the 11th Annual Congress of the European College of Sport Science, Lausanne Switzerland.

29. Franco, S., Rodrigues, J., \& Castañer, M. (2012). Case study 6.3: The behaviour of fitness instructors and the preferences and satisfaction levels of users. In O. Camerino, M. Castañer \& M. T. Anguera (Eds.), Mixed Methods Research in the Movement Sciences (pp. 202-214). Oxon: Routledge.

30. Franco, S., Rodrigues, J., Balcells, M. (2008). Comportamento pedagógico dos instrutores das aulas de grupo de fitness de localizada. Fitness \& Performence Journal, 7 (4), 251-263. https://doi.org/10.3900/fpj.7.4.251.p

31. Franco, S., Simões, V., Alves, S., Vidal, L., \& Silva, C. (2010). Clima nas aulas de Body Pump. Caraterização e comparação entre instrutores com diferentes niveis de experiência profissional $e$ habilitações académicas. Paper presented at the III Congresso Luso-Brasileiro de Educação Física, Desporto e Lazer, Instituto Superior da Maia (ISMAI), Maia, Portugal.

32. Gilbert, W., Trudel, P., Gaumond, S., \& Larocque, L. (1999). Development and aplication of an 


\section{Desenvolvimento e Validação do SOCA-AGF - Aplicação Piloto em Idosos}

instrument to analyse pedagogical content interventions of ice hockey coaches. Sociology of Sport Online, 2. Acedido em janeiro de 2019, (http://physed.otago.ac.nz/sosol/v2i2/v2i2a2.htm)

33. Gomes, I., Chagas, R., \& Mascaranhas, F. (2010). A indústria do Fitness, a mercantilização das práticas corporais e o trabalho do professor de Educação Física: o caso Body Systems. Revista Movimento, $16 \quad$ (4), 169-189. https://doi.org/10.22456/1982-8918.14561

34. Hernández-Mendo, A., Díaz Martínez, F. \& Morales-Sánchez, V. (2010). Construcción de una herramienta observacional para evaluar las conductas prosociales em las classes de educación física. Revista de Psicología del Deporte, 19 (2), 305-318.

35. Hernández-Mendo, A., Blanco-Villaseñor, A., Pastrana, J.L., Morales-Sánchez, V., RamosPérez, F.J. (2016). SAGT: Aplicación informática para análisis de generalizabilidad. Revista Iberoamericana de Psicología del Ejercicio y el Deporte, 11(1), 77-89.

36. Lippke, S., Knäuper, B., \& Fuchs, R. (2003). Subjective theories of exercise course instructors: causal attributions for dropout in health and leisure exercise programmes. Psychology of Sport and Exercise, 4(2), 155-173. https://doi.org/10.1016/S1469-0292(01)00036-X

37. Losada, J.L. \& Manolov, R. (2015). O processo de treinamento básico, treinamento aplicado, mantendo o desempenho de um observador. Qualidade e Quantidade, 49(1), 339-47.

38. Loughead, T. M., \& Carron, A. V. (2004). The mediating role of cohesion in the leader behavior - satisfaction relationship. Psychology of Sport and Exercise, 5, 355-371. https://doi.org/10.1016/S1469-0292(03)00033-5

39. Loughead, T. M., Colman, M. M., \& Carron, A. V. (2001). Investigating the mediational relationship of leadership class cohesion, and adherence in an exercise setting. Small Group Research, 32(5), 558-575.

https://doi.org/10.1177/104649640103200503

40. Mars, H. (1989). Observer reliability: issues and procedures. Champaign. In D. Darst, Z. B. \& V. Mancini (Eds.), Analysing physical education and sport instruction (pp. 53-80). Illinois: Human Kinetics.

41. Murray, D., \& Howat, G. (2002). The relationships among service quality, value, satisfaction, and future intentions of customer at an australian sports and leisure centre. Sport Management Review, 5, 25-43.https://doi.org/10.1016/S14413523(02)70060-0

42. Papadimitriou, D. A., \& Karteroliotis, K. (2000). The service quality expectations in private sport and fitness centers: A reexamination of the factor structure. Sport Marketing Quarterly, 9(3), 157164.

43. Pestana, M. H., \& Gageiro, J. N. (2005). Análise de dados para ciências sociais - A complementaridade do SPSS. Lisboa: Edições Sílabo.

44. Prudente, J., Garganta, J., \& Anguera, M. T. (2004). Desenho e validação de um sistema de observação no Andebol. Revista Portuguesa de Ciências do Desporto, 4(3), 49-65. https://doi.org/10.5628/rpcd.04.03.49

45. Rodrigues, F., Bento, T., Cid, L., Neiva, H., Teixeira, D., Moutão, J., Marinho, D.A., Monteiro, D. (2018). Can Interpersonal Behavior Influence the Persistence and Adherence to Psysical Exercise Practice in Adults? A Systematic Review. Frontiers in Psychology, 9(2141). https://doi.org/10.3389/fpsyg.2018.02141

46. Rodrigues, F., Neiva, H., Marinho, D.A., Mendes, P., Teixeira, D.S., Cid, L., Monteiro, D. (2019). Assessing Need Satisfaction and Frustration in Portuguese Exercise Instructors: scale validity, reliability and invariance betwen gender. Cuadernos de Psicologia del Deporte, 19(1), 22340.

47. Rodrigues, J. (1997). Os treinadores de sucesso. Estudo da influência do objetivo dos treinos e do nível de prática dos atletas na atividade pedagógica do treinador de voleibol. Cruz Quebrada. Lisboa: Edições Faculdade Motricidade Humana.

48. Santos, F. M., Fernandez, J., Oliveira, M. C., Leitão, C., Anguera, M.T., \& Campaniço, J. (2009). The pivot player in handball and patterns 


\section{Dias, I., Franco, S., Ramos, L., Simões, V.}

detection - Instrument. Motricidade, 5, 29-36. https://doi.org/10.6063/motricidade.5(3).193

49. Sarmento, P. (2004). Pedagogia do Desporto e Observação. Cruz Quebrada. Lisboa: Edições Faculdade de Motricidade Humana.

50. Sarmento, P., Veiga, A. L., Rosado, A., Rodrigues, J., \& Ferreira, V. (1998). Pedagogia do Desporto: instrumentos de observação sistemática da Educação Física e Desporto. Cruz Quebrada. Lisboa: Edições Faculdade de Motricidade Humana.

51. Theodorakis, N., Alexandris, K., Rodriguez, P., \& Sarmento, P. J. (2004). Measuring customer satisfaction in the context of health clubs in Portugal. International Sports Journal, 8(1), 44-5

52. Wininger, S. R. (2002). Instructors' and classroom characteristics associated with exercise enjoyment by females. Perceptual and Motor Skills, 94, 39598. https://doi.org/10.2466/pms.2002.94.2.395 